\title{
Quality of Life of Differentiated Thyroid Cancer Survivors On/Off Treatment with Eltroxin, Sohag, Egypt.
}

\section{Rasha Abdel Hameed Ali, Wafaa Abdel Hameed Elsayed, Raafat Abdel Aal Bekheet Mohamed}

Public Health \& Community Medicine, Faculty of Medicine, Sohag University, Sohag, Egypt

\begin{abstract}
Background: Although differentiated thyroid cancer (DTC) has a low disease-specific mortality, this does not reflect their patients' personal experience of the disease. Health related quality of life (QOL) of thyroid cancer survivors have not been well studied in Arab countries especially in Egypt. Objective: This study aimed to assess QOL of DTC patients during and after withdrawal of treatment with L thyroxin. Method: a case control study was conducted in Sohag Institute of Cancer, Egypt, using the 36- SF QOL questionnaire among 89 DTC patients and 100 cancer free persons matched by age, sex, educational and socioeconomic level. QOL of DTC patients were assessed twice during treatment with Eltroxin (base line QOL) and after three weeks of its withdrawal. Results: the mean age (SD) of the studied participants was 39 (1.4) years. The majority were female, rural residents and illiterate $(70.7 \%, 88.7 \%$ and $85.3 \%$ respectively). They reported lower base line QOL in six of the eight domains of 36- SF questionnaires. Physical Component summary (PCS) and Component Summary (MCS) mean score were 75.7 (22.0) and 67.3 (23.2). Significantly reduced QOL of the studied participants was reported after LT4 withdrawal. Both PCS and MCS were significantly higher among patients whose age was lower than 45 years, and those who were followed up for more than three years. Conclusion: DTC survivors in Sohag, Egypt, showed decreased quality of life which became worse during every surveillance cycle as a result of stopping LT4 treatment and older DTC survivors suffers a lot and need better treatment and surveillance regimen.
\end{abstract}

Key word: DTC survivors, $Q O L, L T 4$ withdrawal, predictors of $Q O L$

Corresponding author: Rasha AbdElhameed Ali Mohamed Email: rashaali815@yahoo.com

\section{Introduction}

Thyroid cancer is the most common endocrine malignancy particularly its differentiated type (DTC) which showed the fastest increasing incidence of all malignancies over the past three decades. ${ }^{1}$ Arising from thyroid follicular epithelial cells, DTC includes papillary carcinoma, follicular carcinoma, and less frequently found the Hurthle cell carcinoma. ${ }^{2}$ According to the National Cancer Registry Program, differentiated thyroid cancer is the fifth most frequent cancer in Egyptian females accounting for 3.6\% of all malignancy in women. ${ }^{3}$ Thyroid malignancies are characterized by a wide range of symptoms which may induce both emotional and physical discomfort and may affect all aspects of patients' life. $^{4}$

After initial diagnosis, patients with differentiated thyroid carcinoma (DTC) are treated by total thyroidectomy and radioactive iodine ablation therapy [4]. This is followed by lifelong treatment with Levothyroxine (LT4) which suppresses Thyroid Stimulating Hormone (TSH) in order to suppress the growth of any remaining thyroid cells. ${ }^{4-5}$

DTC generally is a slow growing disease with excellent 10 and 20 year survival 
rates of $90 \%$ and $60 \%$ respectively. Despite these good survival rates, survivors must undergo long-term surveillance to evaluate local recurrence and/or metastatic disease, which is done every 6 months in the first two years and then yearly for three more years, using blood tests thyroglobulin measurement, $\mathrm{I}_{131}$ whole body scan and neck ultrasound. ${ }^{6}$ This is done after induction of clinical hypothyroidism state (by stopping LT4 intake for three weeks) as radioactive iodine uptake is enhanced by TSH level more than 30mU/L. The need for this lifetime management of thyroid functions after surgery and testing procedures used for disease surveillance results in major disruption of patient's life. $^{7}$

The quality of life (QOL) in medicine means physical, mental and social wellbeing, as well as the patients' ability to perform ordinary tasks in their daily existence. According to the World Health Organization (WHO), the QOL is defined as the perception of people of their position in life, in relation to their goals and value system which they have accepted and incorporated into the perspective of decisions that they make (WHO 1998). ${ }^{8}$ Accordingly, many factors interact and affect QOL such as physical health, social relationships, mental and psychological state, level of independence, and is associated with the environment in which the person is living. ${ }^{9}$

There is limited information about the medium to long-term QOL of DTC patients in Egypt, as to our knowledge, no previous studies were done to assess QOL of such patients. The aim of this study was to assess and compare QOL of DTC survivors during LT4 administration and after 3 weeks LT4 withdrawal in addition to investigate predictors of QOL among these patients.

\section{Method}

Study design: A case control study was conducted from January $1^{\text {st }}$ to July $30^{\text {th }} 2018$, among patients previously diagnosed with DTC who were followed in Nuclear Medicine Unit of Sohag National Cancer Institute. We assessed their quality of life in two occasions (during administration of LT4 and after 3 weeks of LT4 withdrawal). These patients were compared to control group of 100 volunteers.

Setting: Sohag Institute of Cancer, which provides free medical services since 1999 to all cancer patients from Upper Egypt, including residents of Sohag, Qena, Red Sea, Aswan and Luxor governorates. This health center provides diagnostic health services in addition to surgical management, chemotherapy and radiotherapy to all cancer patients. DTC patients are followed in this health center by nuclear medicine physicians using radioactive Iodine scanning.

Study participants: In order to participate, subjects must have met the following inclusion criteria: should be 18 years or older; who have been previously diagnosed with and cured from DTC and initial therapy was finished at least 1 year before study inclusion. Subjects were excluded from the study if: 1) were having a benign thyroid nodule or other thyroid cancer such as anaplastic thyroid cancer and medullary thyroid cancer 2)their age were less than 18 years and 3 ) if they had history of other types of head and neck cancer. Consequently100 DTC survivors who met the inclusion criteria agreed to participate and were successfully enrolled in the study.

The control group included 100 volunteers who were matched by age, gender, educational level, diabetes and hypertension status, as much as possible

Table (1) Socioeconomic and clinical characteristics of the studied 89 DTC survivors in Sohag, Egypt

No
$(n=89)$$\quad \%$




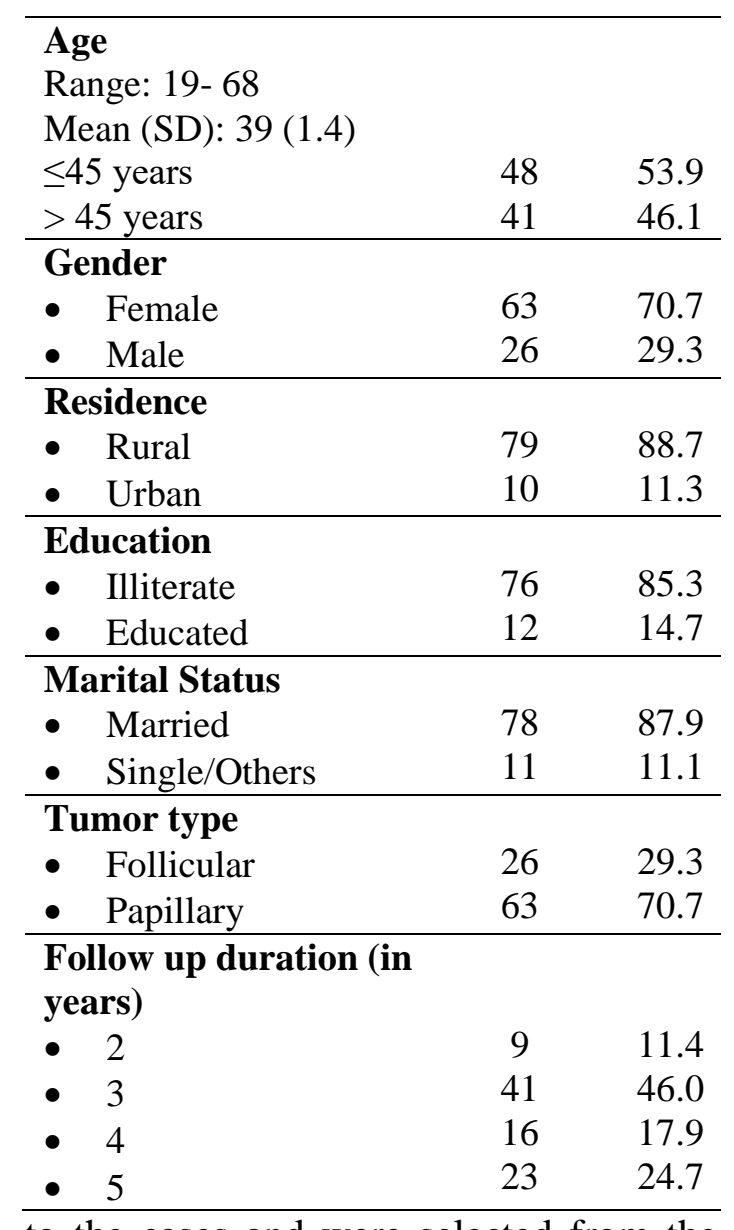

to the cases and were selected from the relatives of the patients who accompanied them in their visits to the health center.

Data collection procedure: Face-to-face interview with each participant was conducted by the researcher in order to complete the two sections questionnaire designed for this study. The first section of the questionnaire included sociodemographic data (age, gender, educational level, residence, marital status), type of thyroid cancer whether follicular or papillary, duration after surgery (for the cases). The second section included The SF-36 (Arabic version) which was used to evaluate the quality of life of thyroid cancer patients in the present study. The designed questionnaire was self-administered but completion of the questionnaires of noneducated patients was done with help of Table (2) comparison between QOL of the studied DTC survivors onloff treatment with Eltroxin and QOL of the control group Sohag, Egypt Which domain is affected the most in during compared to after withdrawal?? the researcher. The SF-36 QOL part of the designed questionnaire were completed twice for each patient, At first during their usual administration of LT4( base line QOL), then patients were instructed to stop taking their medications( LT4) for three weeks to enhance TSH level and the TSH level is tested and when reached above 30 the patients were asked to fill the QOL questionnaire again.

Study instruments: We used validated Arabic version of SF-36 QOL questionnaire. ${ }^{10}$ The SF-36 questionnaire is a generic questionnaire which determines the overall health-related QOL during the previous 30 days. It produces an 8-scale profile (physical functioning $[\mathrm{PF}]$, general health perceptions $[\mathrm{GH}]$, vitality $[\mathrm{VT}]$, rolephysical $[\mathrm{RP}]$, bodily pain $[\mathrm{BP}]$, roleemotional [RE], social functioning [SF], and mental health $[\mathrm{MH}]$ ) of health that can be used to calculate summary mental and physical health composite scores. Each scale of scores is transformed to a scale of 0 to 100; higher scores indicate better quality of life, and lower scores indicate worse quality of life. Next, the Physical Component Summary (PCS) and Mental component Summary (MCS) scores were summarized and compared.

\section{Statistical analysis}

SPSS software for Windows (version 22.0, Chicago, IL, USA) was used for statistical analysis of the data. Sample characteristics were summarized as numbers and percentage for categorical variables and means and SD for numerical variables. Mean comparisons of QOL scores between different sub-groups (gender, age, educational level, marital status, tumour type and follow up duration) were performed using MannWhitney U - tests.

\section{Ethical consideration}




\begin{tabular}{|c|c|c|c|c|c|c|}
\hline \multirow{3}{*}{ QOL domains } & \multicolumn{2}{|c|}{$\begin{array}{c}\text { During Eltroxin } \\
\text { treatment }\end{array}$} & \multirow{3}{*}{$P$ value } & \multicolumn{2}{|c|}{$\begin{array}{c}\text { After Eltroxin } \\
\text { withdrawal }\end{array}$} & \multirow{3}{*}{$P$ value } \\
\hline & Cases & Control & & Cases & Control & \\
\hline & Mean (SD) & $\overline{\text { Mean (SD) }}$ & & Mean (SD) & $\overline{\text { Mean (SD) }}$ & \\
\hline Physical functioning & $81.9(22.6)$ & $86.7(21.8)$ & 0.006 & $52.3(21.0)$ & $86.7(21.8)$ & $<0.001$ \\
\hline General health & $59(24.8)$ & $79.7(26.6)$ & 0.002 & $26.9(22.1)$ & $79.7(26.6)$ & $<0.001$ \\
\hline Role limitation physical & $79(27.1)$ & $88.5(25.0)$ & 0.04 & $1.9(22.0)$ & $88.5(25.0)$ & $<0.001$ \\
\hline Bodily Pain & $82.4(20.7)$ & $86(2.2)$ & 0.04 & $58.5(20.0)$ & $86(2.2)$ & $<0.001$ \\
\hline PCS & $75.7(22.0)$ & $86.1(2.1)$ & 0.001 & $35.1(20.0)$ & $86.1(2.1)$ & $<0.001$ \\
\hline Emotional well-being & $60.5(19.1)$ & $65.5(19.3)$ & 0.08 & $28.5(22.0)$ & $65.5(19.3)$ & $<0.001$ \\
\hline Role limitation- Emotional & $73.3(24.4)$ & $86.5(28.0)$ & 0.01 & $1.86(12.0)$ & $86.5(28.0)$ & $<0.001$ \\
\hline Energy/Fatigue & $57.8(22.4)$ & $63.5(25.6)$ & 0.02 & $26.7(17.0)$ & $63.5(25.6)$ & $<0.001$ \\
\hline Social functioning & $77.5(23)$ & $83.0(2.2)$ & 0.08 & $51.4(21.0)$ & $83(2.2)$ & 0.001 \\
\hline MCS & $67.3(23.2)$ & $79.8(2.2)$ & 0.04 & $27.1(18.0)$ & $79.8(2.2)$ & 0.0001 \\
\hline
\end{tabular}

Prior to data collection, ethical approval was obtained from Scientific Research Ethics Committee of the Faculty of Medicine, Sohag University. In addition, the researchers explained the purpose of the study to all the participants ensuring strict confidentiality and anonymity of all the collected data.

\section{Results}

Out of 100 patients successfully recruited in the current study, 89 cases (11 questionnaires were incomplete) and 100 control persons completed the questionnaire. Ranging between 19- 68 years, the mean age $(\mathrm{SD})$ of the studied patients was 39 (1.4) years. More than half of them were younger than 45 years (52.7\%) and more than two thirds of them were females $(70.7 \%)$. Sociodemographic and clinical data of the studied subjects are detailed in table (1).

Table (2) shows QOL of the studied DTC survivors during treatment with Eltroxin and the QOL of the control group subjects. Compared with the control group, DTC cases reported significantly lower QOL in most of the eight domains of $36 \mathrm{SF}$ questionnaires ( $\mathrm{P}$ value less than 0.05 except for emotional wellbeing and social functioning domains). For DTC patients, the highest mean scores were in scales of Physical Functioning (PF), Bodily Pain (BP) and Role limitation Physical (RP) (81.9 (22.6), 82.4 (2.7) and 79 (27.1) respectively), eventually
Physical Component summery (PCS) mean score was 75.7 (22). The lowest mean scores were those of Energy / Fatigue (57.8(22.4)) and Emotional WellBeing scales (60.5 (19.1)) Mental Component Summery (MCS) score was 67.3 (23.2).

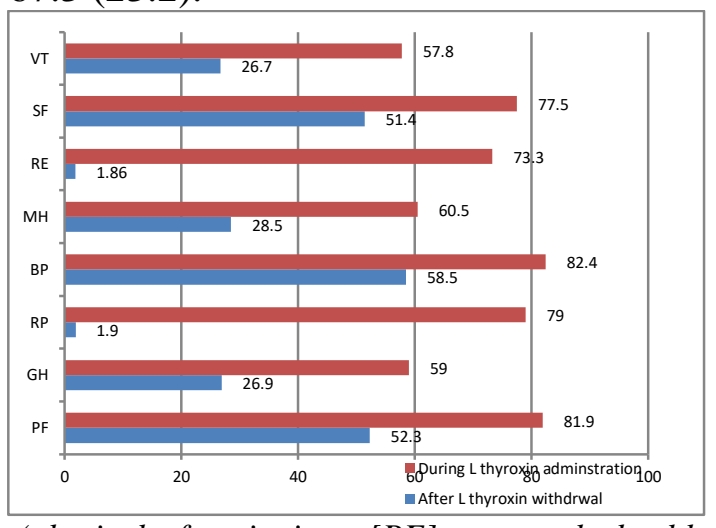

(physical functioning [PF], general health perceptions [GH], vitality [VT], role-physical $[R P]$, bodily pain $[B P]$, role-emotional [RE], social functioning $[S F]$, and mental health $[M H])$.

Figure (1): Comparison between mean score of each domain of baseline QOL of the studied DTC survivors and after three weeks of T4 withdrawal in Sohag, Egypt.

Figure (1) display comparison between mean score of each domain of QOL of the studied DTC survivors during period of treatment with LT4 and after three weeks of its withdrawal. Patients reported significantly worse scores on almost all domains of the QOL after LT4 withdrawal ( $\mathrm{P}$ value<0.05), particularly, large differences were found for Role 
Physical (mean score 79 Vs. 1.9) and Role Emotional (mean score 73.3 Vs. 1.86).

Table (3) shows relation between physical component summery PCS and mental component summery MCS of QOL of DTC survivors and sociodemographic and clinical factors. PCS and MCS were significantly higher among patients whose age was lower than 45 years, and also for those who were followed up for more than three years. No significant effect of gender, educational level, residence and marital status on QOL of the studied subjects was detected.

Results of correlation ( $\mathrm{r}$ ) analysis between independent variables (age and duration of follow up) and MCS and PCS of the studied DTC survivors during treatment with LT4 and after three weeks of its withdrawal are displayed in table (4). Significant negative correlation was detected between both PCS and MCS and the age of the patients ( $\mathrm{P}$ value $<0.05$ ) during treatment with LT4 and after its withdrawal. Correlation between duration of follow up and PCS and MCS failed to reach significance.

\section{Discussion}

Although DTC has a Low diseasespecific mortality, this does not reflect their patients' personal experience of the disease. ${ }^{11}$ Health related QOL of thyroid cancer survivors have not been well studied in Arab countries especially in Egypt. We assessed QOL of DTC survivors and we compared baseline QOL of our patients to QOL of a matched control group. We also compared QOL of the studied subjects during treatment with eltroxin to their QOL after three weeks of its withdrawal in preparation for $\mathrm{I}_{131}$ procedures and thyroglobulin testing hoping to identify the magnitude of reduction in life quality.

In line with previous researches ${ }^{12-20}$, the studied subjects reported lower baseline QOL in all eight domains of the SF-36 QOL compared to the control group. In agreement with Juxian et al., $2017^{13}$ six of these eight domains were significantly lower among cases especially (general health and physical functioning). Poor base line QOL of DTC survivors despite is still a matter of debate. Some studies suggested changes in other thyroid hormone metabolites, lack of endogenous T3 secretion by the thyroid and interference by autoimmune processes. ${ }^{21,22}$ Furthermore, Megan et al., found that thyroid cancer survivors report an overall similar QOL to the survivors of colon, glioma and breast which carry worse 5- year survival rates. ${ }^{21}$ Also the lower score may be associated with struggles and fears related to the cancer experience. ${ }^{13}$ This support our result and indicates that prognosis alone cannot predict QOL as routine surveillance for recurrence in DTC patient is a regular reminder of their disease and enhance constant fear of recurrence, metastasis or fear of a second cancer.

Except for general health domain results which was comparable to Juxiang et al., the poor baseline QOL was much worse than the mentioned studies and domains affected markedly were that of Energy/ Fatigue, General Health and Emotional Well-being which barley had a mean score of 60.5 reflecting markedly reduced life quality in both its physical and mental aspects. ${ }^{13}$ This could be explained by factors related to the studied sample, as the majority of the studied patients were poor, illiterate females who resided in rural areas and traveled a long distance to reach this free public health center for follow up session and found difficulty in understanding treatment instructions for optimal compliance.

In the comparison of QOL scores during period of (LT4) administration and the scores after its withdrawal, patients reported significantly worse scores on almost all domains of the QOL during LT4 withdrawal, which in line with studies conducted by Rubic et al., 2014 
and Davids et al,.2006. ${ }^{11,23}$ The studied subjects reported that the already poor life quality during LT4 treatment is markedly reduced after LT4 withdrawal, as both PCS and MCS were reduced to the half.

These findings were not surprising as it is well known that hypothyroidism resulted in a wide range of physical and psychological symptoms, such as weight gain, fatigue, constipation, depression and slowed cognition, and the associated distress is high. ${ }^{11,24}$ Moreover, psychological impairment and distress are also linked to initial diagnosis, and fear of recurrence which occur with every time of surveillance. ${ }^{11}$ However, what was striking the magnitude of decrease in QOL after LT4 withdrawal, in particular, large differences were found for Role Physical (mean score 79 Vs. 1.9) and Role Emotional (mean score 73.3 Vs.1.86), this may be explained by sample characteristics as most of the studied patients were poor illiterate females who had to stay on lower doses of (LT4) after reaching TSH threshold waiting for radioactive iodine dose which is sometimes delayed because of funding and transport issues, such conditions elongates the duration of suffering of hypothyroid state and affect their house hold duties and result in marked anxiety and psychological upset.

Among the studied predictors of QOL in DTC survivors, age was found to be important predictive factor, as being older than 45 years significantly decreases QOL of the studied subjects (PCS and MCS $\mathrm{P}$ value $=0.009$ and 0.04 respectively), with significant negative correlation between the age and QOL. This is in line with several studies. ${ }^{14,16,18,19,20,25,26}$ This could be caused by the fact that patients above the age of 45 tolerated the symptoms of pretreatment hypothyroidism and radioiodine treatment less well than other younger patients. ${ }^{12}$
On contrary to another study ${ }^{11}$, gender and marital status did not affect QOL which agree with other studies. ${ }^{19,20}$ Moreover, another study's ${ }^{14}$ findings were in line with the current study as QOL of the studied subjects was not affected by their educational level. One more study ${ }^{25}$ agree with the current study as regard absence of significant effect of marital and work status on QOL.

Although correlation between QOL and duration of follow up failed to reach significance, QoL seems to improve after three years of follow up especially PCS. Although Lincolin et al, $2007^{14}$ disagree with the current findings, this was in line with data from other studies. ${ }^{12,15,25}$

\section{Conclusion}

The current study has shown that DTC survivors in Sohag, Egypt, had decreased long term life quality which became worse during every surveillance cycle as a result of stopping LT4 treatment and their QOL is not associated with gender, marital status, residence or educational level. Older DTC survivors suffer a lot and need better treatment and surveillance regimen.

This study, to our knowledge, is the first study to assess QOL of Egyptian DTC survivors and its predictors. It is also the first study to compare baseline QOL and after LT4 withdrawal hoping to determine QOL of these patients during their different post treatment clinical circuit in order to pay attention to treatment modalities that may reduce their suffering and improve their life quality.

This study has some limitations: at first, we were only powered to statistically detect relatively large effects due to the limited number of study subjects, and second, as the study setting was a public health center where most of the customers were from low socioeconomic class suffering a lot in an overloaded health system in low income country, their QOL 
was markedly affected by their health state .

\section{References}

1- Pagano L, Klain M, Pulcrano $M$, Angellotti G, Pasano F, Salvatore M, Lombardi G, BiondiFollow-up of differentiated thyroid carcinoma. MinervaEndocrinal. 2004, Dec; 29(4):16174.

2- Jemal A, Siegel R, Ward E, Hao Y, Xu J, Thun MJ. Cancer statistics, 2009.CA Cancer J Clin.2009; 59:225-49.

3- RashaAbdElhameed Ali Mohamed, RaafatAbdElaalBackeet,WafaaAbdElhamee dElsaayed. Risk factors of differentiated thyroid cancer in Sohag, Egypt.Int. J. Curr. Res. Med. Sci. (2016). 2(11): 27-34

4- Pacini F, Castagna MG, Brilli L, Pentheroudakis G \& ESMO Guidelines Working Group: Thyroid cancer: ESMO Clinical Practice Guidelines for diagnosis, treatment and follow-up. Ann Oncol 2010; 21:214-219.

5- Nix P, NicolaidesA\&Coatesworth AP: Thyroid cancer review2: Management of Differentiated Thyroid Cancers. Int $\mathbf{J}$ ClinPract 2005; 59:1459-1463.

6- Gamper EM, Wintner LM, Rodrigues M, Buxbaum S, Nilica B, Singer $S$, et al. Persistent quality of life impairments in differentiated thyroid cancer patients: results from a monitoring programme. Eur $\mathrm{J}$ Nucl Med Mol Imaging. 2015; 42(8):1179- 1188.

7- Duan H, Gamper E, Becherer A, Hoffmann M. Quality of life aspects in the management of thyroid cancer. Oral Oncol. 2015; 51(6):S1-S5.

8- World Health Organization (WHO). WHOQOL. The World Health Organization Quality of Life Assessment (WHOQOL): development and general psychometric properties. SocSci Med 1998; 46(12): 156985.

9- Singer S, Lincke T, Gamper $\mathrm{E}$ et al. Quality of life in patients with thyroid cancer compared with the general population. Thyroid 2012; 22(2): 117-24

10- Saud Abdul-Aziz Al Abdulmohsin, Stephen Joel Coons, Jolain R. Draugalis, Ron D. Hays, Translation of the RAND 36- item Health Survey 1.0 (aka SF- 36) into Arabic.RAND, 1997
11- Rubic M, Kuna SK, Tesic V, et al. The most common factors influencing on quality of life of thyroid cancer patients after thyroid hormone withdrawal. Psychiatr Danub.2014; 26Suppl 3:520-7.

12- Hoftijzer, H.C., Heemstra, K.A., Corssmit, E.P. et al. (2008) Quality of life in cured patients with differentiated thyroid carcinoma. Journal of Clinical Endocrinology and Metabolism, 93, 200-203

13- Juxiang Gou, MSa, , Wenjie Cheng, MDa, , Jianyong Lei, MDa, , Qian Pan, MDa, , Wei You, MDa, , Ming Cai, MDa, ,Huairong Tang, MDb,*, Yali Lei, MSb, Zhihui Li, MDa, , 13. Rixiang Gong, MDa, ,Jinqiang Zhu, MDa. Health-related quality-of-life assessment in surgical patients with papillary thyroid carcinoma.A single-center analysis from Mainland China. Medicine (2017) 96:38 14- Lincoln G. L. Tan, MBBS, MRCS, MMed; Luo Nan, PhD; Julian Thumboo, MBBS, MRCP, MMed, FAMS, FRCP; Felix Sundram, MBBCh, MSc DMRT; Luke K. S. Tan, MBBS, FRCS, MMedSci, FAMS(ORL). Health-Related Quality of Life in Thyroid Cancer Survivors. Laryngoscope (2007), 117: 507-510

15- E.T. Massolt, M. van der Windt, T.I.M. Korevaar, B.L.R. Kam, J.W. Burger, G.J.H. Franssen, I. Lehmphul, J. Kohrle, W.E. Visser and R.P. Peeters. Thyroid hormone and its metabolites in relation to quality of lifein patients treated for differentiated thyroid cancer. Clinical Endocrinology (2016), 85, 781-788

16- David Taïeb1, KarineBaumstarckBarrau, FrédéricSebag, Cécile Fortanier, Catherine De Micco, Anderson Loundou, Pascal Auquier, Fausto F Palazzo, Jeanfrançois Henry and Olivier Mundler. Heathrelated quality of life in thyroid cancer patients following radioiodine ablation. Health and Quality of Life Outcomes 2011, 9:33

17- Megan K. Applewhite, Benjamin C. James, Sharone P. Kaplan, Peter Angelos, Edwin L. Kaplan, Raymon H. Grogan,Briseis, Aschebrook-Kilfoy. Quality of Life in Thyroid Cancer is Similar to That of Other Cancers with Worse Survival. World J Surg (2016) 40:551-561

18- Almeida JP, Vartanian JG, Kowalski LP: Clinical predictors of quality of lif in patients with initial differentiated thyroid cancers.

Vol. 38 No. 3 
Arch

Head Neck Surg 2009, 135:342-346.

19- Pelttari H, Sintonen H, Schalin-Jantti C, Valimaki MJ: Health-related quality of life in long-term follow-up of patients with cured TNM Stage I or II differentiated thyroid carcinoma. ClinEndocrinol (Oxf) 2009, 70:493-497.

20- Tagay S, Herpertz S, Langkafel M, Erim Y, Bockisch A, Senf W, Gorges R: Healthrelated Quality of Life, depression and anxiety in thyroid cancer patients. Qual Life Res 2006, 15:695-703

21- Carta, M.G., Loviselli, A., Hardoy, M.C. et al. (2004) The link between thyroid autoimmunity (antithyroid peroxidase autoantibodies) with anxiety and mood disorders in the community: a field of interest for public health in the future. BioMed Central Psychiatry, 4, 25. 22. Watt, T., Hegedus, L., Bjorner, J.B. et al. (2012) Is thyroid autoimmunity per se a determinant of quality of life in patients with autoimmune hypothyroidism? European Thyroid Journal, 1, 186-192.
22- Davids T, Witterick I J, Eski S, Walfish PG \& Freeman J L: Three-Week Thyroxine Withdrawal: A Thyroid-Specific Quality of Life Study. The Laryngoscope 2006; 116:250- 253

23- Dagan T, Bedrin L, Horowitz Z, et al. Quality of life of welldifferentiated thyroid carcinoma patients. J Laryngol Otol. 2004; 118(7):537-42.

24- SiedKazemRazaviRatki, BabakFallahi, NassimNamiranian , AlirezaEmamiArdekani , Mohsen Saghari , ArashMirabzadeh , ArmaghanFard-Esfahani , DavoodBeiki , Mohammad Eftekhari, FarzanehPooyafard. Factors affecting the quality of life of well-differentiated thyroid carcinoma patients: A cross-sectional study on 435 Iranian patients. Iran J Nucl Med 2016;24(2):92-98

25- Mendoza A, Shaffer B, Karakla D, et al. Quality of life with well-differentiated thyroid cancer: treatment toxicities andtheir reduction. Thyroid 2004; 14:133-140

Table (3) Relation between physical component summery PCS and mental component summery MCS of QOL of DTC survivors and sociodemographic and clinical factors.

\begin{tabular}{|c|c|c|c|c|}
\hline \multirow[t]{2}{*}{ Factors } & PCS & \multirow[t]{2}{*}{ P value } & MCS & \multirow[t]{2}{*}{ P value } \\
\hline & $\begin{array}{l}\text { Mean score } \\
\text { Mean (SD) }\end{array}$ & & $\begin{array}{l}\text { Mean score } \\
\text { Mean (SD) }\end{array}$ & \\
\hline $\begin{array}{l}\text { Age } \\
\leq 45 \\
>45\end{array}$ & $\begin{array}{l}81.3(13) \\
69.2(24.6)\end{array}$ & 0.009 & $\begin{array}{l}30.4 \\
23.2\end{array}$ & 0.04 \\
\hline $\begin{array}{l}\text { Gender } \\
\text { Female } \\
\text { Male }\end{array}$ & $\begin{array}{l}75.7(23) \\
75.9(21.8)\end{array}$ & 0.9 & $\begin{array}{l}67.1(22.8) \\
67.7(24.57)\end{array}$ & 0.9 \\
\hline $\begin{array}{l}\text { Residence } \\
\text { Rural } \\
\text { Urban }\end{array}$ & $\begin{array}{l}76.37(21.5) \\
70.6(26.6)\end{array}$ & 0.4 & $\begin{array}{l}67.6(23.1) \\
64.4(24.8)\end{array}$ & 0.7 \\
\hline $\begin{array}{l}\text { Education } \\
\text { Illiterate } \\
\text { Educated }\end{array}$ & $\begin{array}{l}75(22.9) \\
80.5(16.5)\end{array}$ & 0.5 & $\begin{array}{l}66.6(23.7) \\
71(22)\end{array}$ & 0.5 \\
\hline
\end{tabular}




\begin{tabular}{|l|l|l|l|l|}
\hline $\begin{array}{l}\text { Marital Status } \\
\text { Married } \\
\text { Single ,divorced or wido }\end{array}$ & $76.2(21.8)$ & 0.7 & $68.3(22.3)$ & \\
& $75.8(22.4)$ & & $62.9(29.2)$ & \\
\hline $\begin{array}{l}\text { Tumor type } \\
\text { Follicular } \\
\text { Papillary }\end{array}$ & $55.1(22.1)$ & & & \\
\hline Follow up duration (i) & $84.2(15.6)$ & & $75.7(18.5)$ & \\
years) & $76.2(2)$ & $\mathbf{0 . 0 0 0}$ & & $\mathbf{0 . 0 0 0}$ \\
$\mathbf{2}$ & $55.7(3.3)$ & & $52.1(1.2)$ & \\
$\mathbf{3}$ & $79.6(2.6)$ & & $70.4(2.1)$ & \\
$\mathbf{4}$ & $82.3(1.6)$ & & $73.1(1.9)$ & \\
$\mathbf{5}$ & & & & \\
\hline
\end{tabular}

Table (4): Correlation ( $r$ ) analysis between independent variables (age and duration of follow up) and MCS and PCS of the studied DTC survivors during LT4 administration and after three weeks of its withdrawal.

\begin{tabular}{|c|c|c|c|c|c|c|c|c|}
\hline \multirow[t]{3}{*}{ variables } & \multicolumn{4}{|c|}{ During $L$ thyroxin administration } & \multicolumn{4}{|c|}{$\begin{array}{l}\text { After three weeks of } L \text { thyroxi } \\
\text { withdrawal }\end{array}$} \\
\hline & \multicolumn{2}{|l|}{ PCS } & \multicolumn{2}{|l|}{ MCS } & \multicolumn{2}{|l|}{ PCS } & \multicolumn{2}{|l|}{ MCS } \\
\hline & $\mathbf{r}$ & $P$ value & $\mathbf{r}$ & $P$ value & $\mathbf{r}$ & P value & $\mathbf{R}$ & P value \\
\hline Age & -0.265 & 0.012 & -0.0184 & 0.008 & -0.285 & 0.007 & -0.285 & 0.007 \\
\hline $\begin{array}{l}\text { Duration } \\
\text { follow up }\end{array}$ & -0.012 & 0.9 & 0.022 & 0.8 & -0.018 & 0.8 & 0.037 & 0.7 \\
\hline
\end{tabular}

